\title{
Control-Oriented Modeling of Ionic Polymer-Metal Composite Enabled Hydrogen Gas Production
}

\author{
Tushar Nagpure, Zheng Chen* \\ Department of Electrical Engineering and Computer Science \\ Wichita State University \\ Wichita, KS 67260, USA
}

\begin{abstract}
Ionic polymer-metal composite (IPMC) is an electro-active polymers which exhibits the property of ion migration under the application of external electric field. This property of IPMC along with the ability to operate in aqueous environment is useful in dissociating chemical bond in water $\left(\mathrm{H}_{2} \mathrm{O}\right)$, and produce hydrogen $\left(\mathrm{H}_{2}\right)$ and oxygen $\left(\mathrm{O}_{2}\right)$ gases. In this paper, a control-oriented model of IPMC as an electrolyzer is presented. By equating the thermodynamic and electrochemical equations of the system, a linear relationship between flow rate of hydrogen generation and the source current is derived. A nonlinear state-space model is then used to capture the source current related to the voltage input. The model has been verified with the experimental data. Temperature-dependent effect and energy-conversion efficiency have been investigated, which shows the need of state feedback control for achieving high energy conversion efficiency. Based on a linearized model, a linear quadratic regulator (LQR) is designed to optimize the control performance. Experimental results have shown that the output performance of LQR is better than that of PID. It has also been validated that the controloriented model is useful in optimal control of IPMC electrolyzer.
\end{abstract}

Keywords: Ionic polymer-metal composites; Water Electrolysis; Hydrogen Production; Modeling; Linear Quadratic Regulator;

\footnotetext{
*Corresponding author, Tel: +1-316-978-7391; fax: +1-316-978-3984.

Email addresses: tcnagpure@wichita.edu (Tushar Nagpure), zheng.chen@wichita.edu (Zheng Chen )
} 


\section{Introduction}

Increase in the dependency on non-renewable energy sources has led to the concern on it's degrading impact on the environment. Majority of it is consumed by the transportation sector. Also, with the gradual decrease in the petroleum based fuel resources over the period of time, it has become necessary to search for alternative fuel options [1]. Therefore research on minimum carbon dioxide emission technologies has been intensified. And the solution is found out to be in the use of vehicles powered by electricity. This has led to the challenges in storage and utilization of electricity, which requires novel and clean methods. In present situation, the electrical vehicles are powered by two technologies, i.e. battery and hydrogen. Battery powered electric vehicle offers low cost per unit energy consumption. However, it suffers from the drawback of slow charging period, which is not ideal for transportation sector. Comparatively, hydrogen as a fuel offers faster refueling time, and can serve higher payloads. Hydrogen $\mathrm{H}_{2}$ gas is one of the clean and green options available among alternative sources such as solar, wind, tidal energy, etc.

Hydrogen has a gas density of $0.0899 \mathrm{~kg} / \mathrm{Nm}^{3}$, which is 15 times lighter than air. It has higher heating value (HHV) of $144 \mathrm{MJ} / \mathrm{kg}$ that is twice to three times more than methane and gasoline, respectively [2]. This makes Hydrogen have the highest mass energy density. When used in combination with other alternative sources of energy such as solar and wind, the hydrogen battery can form a highly efficient energy storage system. Even though hydrogen has abundant availability in nature, implementation of hydrogen as a fuel is disrupted by the fact that it is not available in a stable molecular state. It is mainly extracted from fuel sources, such as coal gasification, natural gas reformation and water electrolysis $[2,3]$. In commercial hydrogen production, 3-4\% comes from electrolysis processes [4].

Ionic polymer-metal composite (IPMC) is an electro-active polymer that exhibits the property of sensing and actuation effects [5]. It consists of an ion exchange membrane, such as Nafion, coated with platinum electrode. Nafion is permeable to the flow of ions, however it is impermeable to the molecules of considerable size such as hydrogen and oxygen molecules [6]. When electrical field is applied to the membrane, the positive surface becomes anode and the negative surface becomes cathode. When the polymer is perfectly hydrated and the magnitude of the electric field is high, it dissociates the water molecule at anode and produces free negative oxygen ions and positive 
hydrogen ions. The oxygen ions recombine with with each other to produce stable diatomic hydrogen molecule. The hydrogen ions passes from anode to cathode. At cathode these free ions combine with the electrons to produce diatomic hydrogen molecule. This diatomic hydrogen molecule is stable in nature and can be stored and used as a fuel. Due to the ion transportation, the IPMC swells in cathode side and shrinks in anode side, which leads to a bending effect [5]. Figure 1 shows the mechanism of both mechanical actuation and water electrolysis. IPMC electrolyzer is advantageous for the following reasons: It has a conductive material between anode and cathode which provide ionic channels to keep activation voltage low. Free sodium and calcium ions act as charge carriers in the membrane, so electrolysis can be done in tap water as well. Platinum electrode made IPMC is chemically stable in water [7]. IPMC has been used for water electrolysis generators by many research groups $[7,8]$.

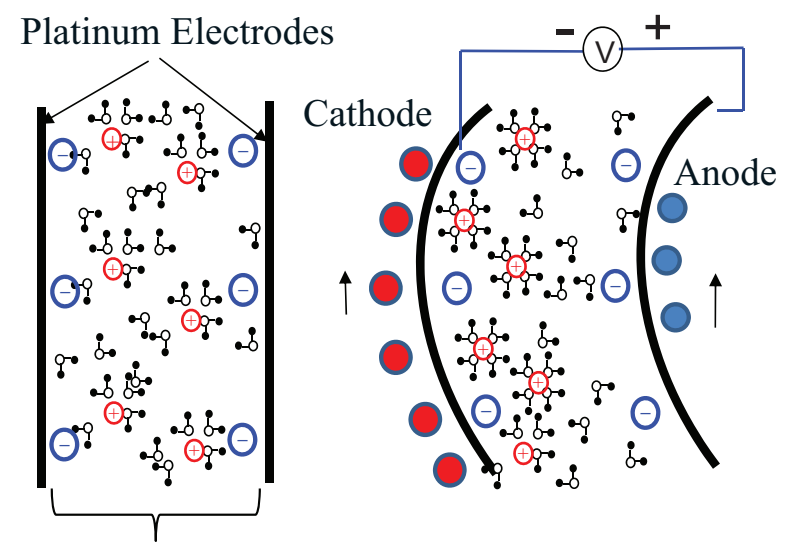

Ion exchange membrane

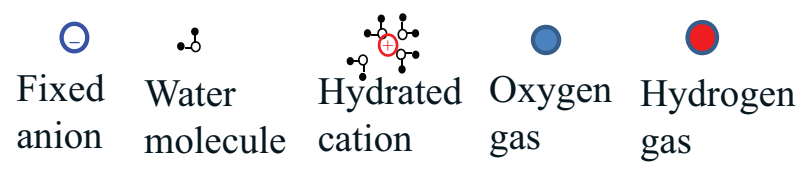

Figure 1: Mechanism of IPMC enabled water electrolysis.

The Nafion based IPMC membrane are highly durable because ionomer as a catalytic layer acts as a binder to provide mechanical stability. Compared to the PEM, IPMC promote proton transport from the concentrated catalyst layer to the membrane. This enhances overall efficiency by reducing ohmic 
losses. However it exhibits highly nonlinear properties. The doping concentrations, structure of the membrane, type of free ions and water conditions highly influence the internal parameters of IPMC for electrolysis. Therefore, for the given sets of input voltage the flow rate of hydrogen gas production varies. Hydrogen gas is stored in high pressure tanks. The high pressure physical storage of hydrogen is achieved with the help of compressors. Hydrogen gas at 1 bar and constant flow-rate act as an input for compression. Given the unpredictable behavior IPMC due to its non-linearity, it is necessary to assure that the output of IPMC electrolyzer is at a constant flow-rate. Therefore demonstrations are needed for control of the flow rate of hydrogen gas

In this context, the paper presents a study on modeling and control of the electrolysis using IPMC. First, it derives a linear relation between the flow rate of hydrogen gas and DC current required for work done based on the electrochemical fundamentals. Second, a nonlinear partial differential equation is introduced to capture the ion transportation dynamics and a nonlinear circuit model is used to capture the impedance of the IPMC electrolyzer. Third, a nonlinear state space model is derived based on the circuit model and electrochemical fundamentals. Fourth, the model obtained is verified by conducting experiments on electrolysis of water to produce hydrogen using IPMC. Fifth, the temperature-dependent effect has been investigated and the energy efficiency of the system is computed by evaluating the electrical energy input to the system and calorific value of hydrogen gas produced. The evidence has been shown that a state feedback control is needed to maintain a high energy conversion efficiency under temperature variations and other disturbances. Last, the model is reduced to a linear model. Based on that, a linear quadratic regulator (LQR) control is designed and implemented in real time. It has also shown that the model-based LQR control is better than a model-free PID control in terms of tracking a desired hydrogen gas flow rate with minimum energy consumption.

The paper is organized as follows: the electrochemical fundamentals are described in Section 2. The ion transportation dynamics is introduced in Section 3. The nonlinear circuit model and control-oriented state space model are discussed in Section 4. Model verification, temperature-dependent effect, and energy conversion efficiency are described in Section 5. Model-based LQR control and implementation are presented in Section 7. Conclusion and future work are discussed in Section 8. 


\section{Electrochemical Fundamentals}

This section describes the actuation voltage for electrolysis and the relation between supply current and hydrogen generation flow-rate.

\subsection{System Thermodynamics}

Electrolysis of water using IPMC produces one mole of hydrogen $\left(\mathrm{H}_{2}\right)$ and a half mole of oxygen.

$$
\begin{gathered}
\text { Anode } 2 \mathrm{H}_{2} \mathrm{O} \rightarrow 4 \mathrm{H}^{+} \mathrm{O}_{2}+4 e^{-} . \\
\text {Cathode } 4 \mathrm{H}^{+}+4 e^{-} \rightarrow 2 \mathrm{H}_{2} .
\end{gathered}
$$

The energy necessary to accomplish electrolysis represents a change in enthalpy of the system. However, with the increase in temperature entropy of the system $(T \Delta S)$ increases and the necessary energy required becomes the energy obtained from difference between the change in enthalpy and the change in entropy, this is called as Gibbs free energy [9].

$$
\Delta G=\Delta H-T \Delta S
$$

where $\Delta G$ is the change in Gibbs free energy per mole, $\Delta H$ is the change in enthalpy per mole, $\Delta S$ is the change in entropy per mole inside the system and $T$ is the temperature of the system. Each molecule of hydrogen $\left(\mathrm{H}_{2}\right)$ is produced by two positive ions of hydrogen and two electrons $(-2 \mathrm{e})$. The charge on each electron is $1.602 \times 10^{-19}$ coulombs. Avogadros number $(\mathrm{Na})$ is defined as the number of molecules per mole, which is given as $6.022 \times 10^{23}$ per mole [10]. Taking the product of electron charge per molecule and Avogadros number gives total molar charge. And since Faraday's constant $(F)$ is the magnitude of electric charge per mole of electrons [11], the total molar charge $q$ is given by:

$$
q=-2 e^{-} N a=-2 F .
$$

Considering IPMC as charged parallel plates, then by definition work per unit charge is.

$$
W=q \Delta V
$$

where $W$ is the work done per mole and $\Delta V$ is the change in applied potential. From the definition of Gibbs free energy from Equation 3, the change in it 
is the energy available to do the work, assuming that it is under a constant pressure and volume condition.

$$
\begin{gathered}
\Delta G=-2 F V^{-}, \\
V^{-}=\frac{\Delta G}{-2 F} .
\end{gathered}
$$

Since, the obtained voltage is negative, it is called reverse voltage $\left(V^{-}\right)$. The significance of this reverse voltage is that the process of electrolysis begins only when applied voltage across electrodes exceeds the magnitude of $\left(V^{-}\right)$. At standard room temperature and 1 atm, reversible potential required is $1.23 \mathrm{~V}$ [11]. Due to variations in the contributing parameters for electrolysis process, the true potential needed for decomposition of water can be as high as $1.6 \mathrm{~V}[12]$.

\subsection{Hydrogen flow rate}

Two electrons circulate through the external circuit for the production of one mole of hydrogen. The charge produced by each mole of hydrogen is given in Equation (4). So, the total charge $Q$ produced by system is equal to molar charge times number of moles produced $N_{m}$. Thus the number of hydrogen moles produced is the fraction of total charge to the molar charge.

$$
Q=q N_{m}
$$

With Equation (4), one can have

$$
N_{m}=\frac{Q}{-2 F}
$$

The number of hydrogen moles produced over the period of time $t$ gives the generated mole rate of hydrogen $\left(M_{2}\right)$. Therefore, the above equation can be expressed in terms of mole rate $\left(\mathrm{M}_{2}\right)$ by dividing both the terms by time,

$$
M_{2}=\frac{Q}{t} \frac{1}{-2 F} .
$$

Since the steady state current $I_{d} c=\frac{Q}{t}$, one can have

$$
M_{2}=\frac{I_{d c}}{-2 F}
$$


Equation (11) describes the flow rate of hydrogen gas in terms of moles per second,which is not measurable in quantity. The mole flow-rate $M_{2}$ is converted in to measurable volumetric flow-rate $H_{2}$.

$$
H_{2}=\frac{I_{d c}}{-2 F} \frac{m}{\rho}
$$

where molar hydrogen mass $(m)$, density $(\rho)$ and $(F)$ are constant values, it can be substituted by constant $(\alpha)$, which is defined as

$$
\alpha=\frac{m \rho}{-2 F} \text {. }
$$

With the appropriate value of constant $\alpha$, the flow-rate of hydrogen gas can be obtained in measurable cubic centimeter per second.

\section{Ion Transportation Dynamics}

While the electrochemical fundamentals capture the hydrogen flow rate in terms of source current, the ion transportation dynamics has not been considered yet. The ion transportation dynamics leads to a model that captures the source current related to a voltage input. In this section, the ion transportation dynamics is described. The governing PDE for charge distribution in an IPMC was first presented in [13] and then used by Farinholt [14] for investigating the actuation and sensing responses. Let $\mathbf{D}, \mathbf{E}, \phi$, and $\rho$ denote the electric displacement, electric field, electric potential, and charge density, respectively. The geometric definitions of an IPMC beam is shown in Fig. 2. The following equations hold:

$$
\begin{aligned}
& \mathbf{E}=\frac{\mathbf{D}}{\kappa_{e}}=-\nabla \phi, \\
& \nabla \cdot \mathbf{D}=\rho=F\left(C^{+}-C^{-}\right),
\end{aligned}
$$

where $\kappa_{e}$ is the effective dielectric constant of the polymer, $F$ is Faraday's constant, and $C^{+}$and $C^{-}$are the cation and anion concentrations, respectively. Since the thickness of an IPMC is much smaller than its length or width, it has been assumed that $\mathbf{D}$ and $\mathbf{E}$ are restricted to the thickness direction ( $x$-direction) only, and from here on drop the boldface notation for these variables. The continuity expression relates the ion flux $J$ (in $x$-direction) to the cation concentration $C^{+}$,

$$
\frac{\partial J}{\partial x}=-\frac{\partial C^{+}}{\partial t}
$$


The ion flux consists of diffusion, migration, and convection terms, and can be expressed as $[13,15]$

$$
J=-\frac{d \kappa_{e}}{F}\left(\frac{\partial^{2} E}{\partial x^{2}}-\frac{F\left(1-C^{-} \Delta V\right)}{R T} E\left(\frac{\partial E}{\partial x}+\frac{F C^{-}}{\kappa_{e}}\right)\right) .
$$

where $d$ is the ionic diffusivity, $R$ is the gas constant, $T$ is the absolute temperature, $\Delta V$ is the volumetric change. With the continuity equation (16), one can derive the nonlinear governing PDE in terms of the electric field,

$$
\begin{aligned}
\frac{\partial^{2} E}{\partial t \partial x} & =d \frac{\partial^{3} E}{\partial x^{3}}-d \frac{F\left(1-C^{-} \Delta V\right)}{R T}\left[\frac{\partial^{2} E}{\partial x^{2}} \cdot E+\left(\frac{\partial E}{\partial x}\right)^{2}\right] \\
& -d \frac{F^{2} C^{-}\left(1-C^{-} \Delta V\right)}{R T \kappa_{e}} \frac{\partial E}{\partial x}
\end{aligned}
$$

It is challenging to analytically solve the nonlinear PDE (18) to obtain the

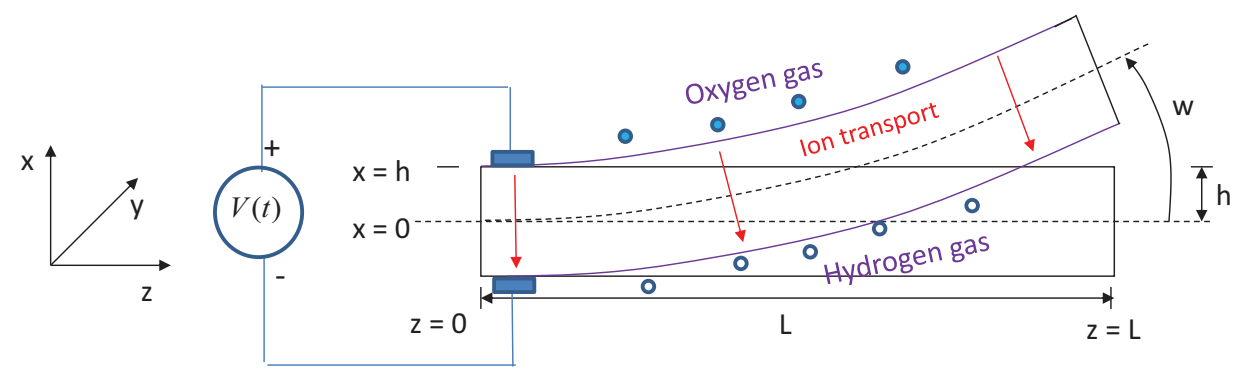

Figure 2: Geometric definitions of an IPMC electrolyzer.

control-oriented model that relates the output source current to the input voltage. An approximation method must to be applied to find the model, which will be described in Section 4.

\section{Control-Oriented Model}

The ion transportation dynamics is governed by PDEs, which is not suitable for control system design. The goal of this research is to derive a controloriented model that the control engineer can use for control design purpose. The approach to modeling of the electrolysis process is based on the identification of equivalent electrical characteristics of IPMC membrane. The 
capacitance and resistance in the circuit model are related to the PDEs described in Section 3. The following descriptions explain the circuit model term by term.

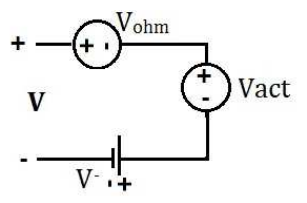

(a)

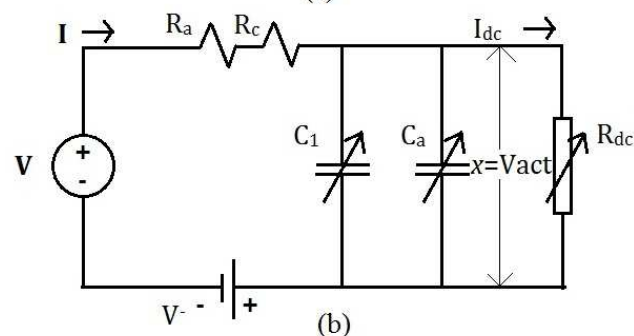

Figure 3: IPMC actuation mechanism for (a) Overpotential model, (b) Equivalent electrical circuit representation of IPMC electrolyzer.

\subsection{Potential model of IPMC}

The losses occurring in the thermodynamic and electrical systems are irreversible and modeled in terms of over potentials inside and on the surface of IPMC cell. According to Carmo et al. [6] the equation used for explaining cell potential $(V)$ is:

$$
V=V^{-}+V_{\text {act }}+V_{\text {ohm }}+V_{\text {trans }}
$$

where $V_{\text {ohm }}$ is the ohmic potential and $V_{\text {act }}$ is the actuation potential. As explained earlier, $V^{-}$is the reversible cell voltage without losses. It is required by the electrolyzer to begin the electrolysis process and mainly depends on the change in Gibbs free energy of the system. From equation (3), Gibbs free energy is temperature dependent. Therefore $V^{-}$also depends on the internal and ambient temperature of the system. The activation potential $V_{\text {act }}$ is the voltage attributed for overcoming electrochemical reaction. The slowness of electrochemical reaction at electrode surface consumes a portion of applied voltage to transfer electrons from electrode. 
The ohmic potential $V_{\text {ohm }}$ is created by resistance from electrode and ionic diffusion. Electrode resistance depends upon the material used for electrode formation. As the resistivity varies with the type of material used, $V_{\text {ohm }}$ changes accordingly. Ionic diffusion is due to opposition to the flow of ions through the IPMC membrane. $V_{\mathrm{ohm}}$ is linear and proportional to the circuit current. $V_{\text {trans }}$ is the mass transport potential and it is the voltage loss due to excess reaction products at electrodes under high pressure and high current densities [16]. It is not significant in this work as the system is operating at $1 \mathrm{~atm}$ and the current density is between $100 \mathrm{~mA} / \mathrm{cm}-200 \mathrm{~mA} / \mathrm{cm}$.

\subsection{Circuit model of IPMC}

As explained in Chen et al. [17], the nonlinear circuit model of IPMC membrane comprises of non-linear capacitance of the membrane $\left(C_{1}\right)$, pseudocapacitance due to electrochemical adsorption $\left(C_{a}\right)$, ion diffusion resistance $\left(R_{c}\right)$, electrode resistance $\left(R_{a}\right)$ and DC resistance of polymer $\left(R_{d c}\right)$. The physics based non-linear circuit model with above parameters is given in Figure 3(b). Comparing the circuit model with cell potential model of IPMC electrolyzer, equations for electrical parameters are derived.

\subsubsection{Ohmic potential of IPMC}

The ohmic potential drop $\left(V_{\text {ohm }}\right)$ is due to the ion diffusion resistance $\left(R_{c}\right)$ and electrode resistance $\left(R_{a}\right)$. IPMC is fabricated with platinum electrodes by chemical deposition process. Due to the platinum deposition over the surface area of the membrane, the concentration of negative ion is high on the electrode compared to the polymer. This results is a Gibbs-Donnan effect which creates electric field in opposition to the applied potential. The potential drop due to the Gibbs-Donnan is represented in terms of ion diffusion resistance $\left(R_{c}\right)$. The electrode resistance $\left(R_{a}\right)$ is the resistance at point of contact between platinum electrode and the membrane. The potential drop due to both of the resistance is termed as ohmic potential and is proportional to the source current.

$$
V_{\mathrm{ohm}}=I\left(R_{a}+R_{c}\right)
$$

\subsubsection{Actuation potential}

The actuation potential $\left(V_{\text {act }}\right)$ is due to the non-linear capacitance of the cell, pseudo-capacitance and non-linear DC resistance. 


\subsubsection{Non-linear capacitance $\left(C_{1}\right)$}

Chen et al. analytically solved the PDEs at steady-state to obtain the nonlinear relationship between the charge and input voltage, which leads to the nonlinear capacitance of IPMC [17]. The nonlinear capacitance captures fundamental physics in IPMC. When voltage is applied across the membrane, current flowing from one electrode to the other creates charge separation at the interface between Nafion and platinum electrode. This effectively creates two capacitors that are connected in series by the polymer called electric double layer. It is expressed in terms of dimension of the membrane and therefore proportional to the surface area $(S)$ and dielectric constant $\left(k_{e}\right)$. Non-linear capacitance is given by:

$$
\begin{gathered}
C_{1}(V)=S k_{e} \frac{\dot{\Gamma}(V)}{\sqrt{2 \Gamma(V)}}, \\
\left.\Gamma(V)=\frac{b}{a}\left(\frac{a V}{e^{a V}-1}-\ln \frac{a V}{e^{a V}-1}\right)-1\right), \\
\left.\dot{\Gamma}(V)=\frac{b}{a}\left(1-\frac{e^{a V}-1}{a V}\right) \frac{e^{a V}-1-a V e^{a V}}{\left(e^{a V}-1\right)^{2}}\right),
\end{gathered}
$$

where $V$ is the applied voltage, $a$ and $b$ are fractions of Faraday constants. When the external potential is applied to IPMC membrane it undergoes physical deformation due to ion movement. In this work the membrane is held rigid across its surface and physical deformation is miniscule, therefore volumetric change in membrane is assumed to be zero. So constants $a$ and $b$ becomes independent of volumetric change in IPMC.

$$
\begin{gathered}
a=\frac{F}{R T} \\
b=\frac{F^{2} C^{-}}{R T k_{e}}
\end{gathered}
$$

Where, $R$ is the gas constant, $T$ is absolute temperature and $C^{-}$is cation concentration.

\subsubsection{Pseudo-capacitance $\left(C_{a}\right)$}

Reversible Faradaic reactions occur on the surface of the electrode. Ions in the Nafion polymer within the electric double-layer transfer some electrons to the atoms of the electrode surface. This is called under potential deposition or 
electrochemical adsorption which results in a Faradaic current $[18,19]$. This charge transfer between the electrode surface and polymer is called pseudocapacitance $\left(C_{a}\right)$. Pseudo-capacitance is $10-100$ times larger than nonlinear capacitance.

$$
\begin{gathered}
C_{a}(V)=\frac{q_{1} S F}{R T} \frac{K_{1} c^{H+} e^{\frac{V F}{R T}}}{\left(K_{1} c^{H+}+e^{\frac{V F}{R T}}\right)^{2}}, \\
K_{1}=\frac{k_{1}}{k_{-1}},
\end{gathered}
$$

where $q_{1}$ is some constant, $k_{1}, k_{-1}$ are the chemical rate constant and $c^{H+}$ is concentration of hydrogen ions in the IPMC. For $\mathrm{H}$ on polycrystalline platinum, $q_{1}=210 \mu \mathrm{C} / \mathrm{cm}^{2}, c^{H+}=10^{-6} \mathrm{~mol} / \mathrm{m}^{3}$ and $K_{1}=4 \times 10^{5}$ [18].

\subsubsection{Non-linear DC resistance $R_{d c}$}

Leakage resistance exists because of the behavior of IPMC as an electrochemical capacitor. In practical case the electrode-solution interface is not ideally polarizable. So upon polarization some potential-dependent Faradaic current passes through the polymer. It can also be stated as the charge transfer resistance in nonlinear capacitance and pseudo-capacitance. Combining this leakage resistance with the ohmic solution resistance, it forms non-linear DC resistance. Assuming that the Butler-Volmer equation describes the dynamic behavior of the reaction, the source current $I_{d c}$ as a function of applied potential $V$ is given as,

$$
I_{d c}=I_{0}\left(e^{\frac{\alpha_{0} F n\left(V-V^{-}\right)}{R T}}-e^{\frac{\alpha_{r} F n\left(V-V^{-}\right)}{R T}}\right),
$$

where $I_{0}$ is the exchange current, $n$ is the number of electrons involved in each reaction and $\alpha_{0}$ and $\alpha_{r}$ are the anode and cathode charge transfer coefficient [20]. It is evident from Equation (28) that the current density is an exponential function of applied potential. Equation (28) can be extended by a Taylor series in terms of $V-V^{-}$. If one defines $G_{d c}=\frac{I_{d c}}{V-V^{-}}$, then $G_{d c}$ is a non-linear conductance, which can be obtained by a series of polynomial functions. A third-order polynomial function gives a good agreement between the measured curve and the calculated curve of $G_{d c}$ conductance for the input voltage $V-V^{-}$.

$$
G_{d c}=m\left|\left(V-V^{-}\right)^{3}\right|+n\left|\left(V-V^{-}\right)^{2}\right|+o\left|\left(V-V^{-}\right)\right|+p,
$$


where $m, n, o, p$ are the coefficients. The nonlinear resistance is then given by $R_{d c}=1 / G_{d c}$.

From Figure (3), the solution for actuation potential can be obtained for steady state condition [21].

$$
V_{c}=V_{c, s s}+V_{c, t r}
$$

where $V_{c, s s}$ is the steady-state voltage response of capacitor branch and $V_{t, t r}$ is transient response. The voltage response of the capacitor is given as:

$$
\begin{aligned}
V_{c} & =\frac{V-V^{-}}{\left(1+\frac{\left(R_{a}+R_{c}\right)}{R_{d} c}\right)}\left(1-e^{t / \tau}\right), \\
\tau & =\frac{\left(R_{a}+R_{c}\right) R_{d c}}{R_{d c}+R_{a}+R_{c}}\left(C_{1}+C_{a}\right) .
\end{aligned}
$$

\subsection{Nonlinear State-Space Model}

Considering physics based the nonlinear circuit model given in Figure 3, by applying Laplace Transformation to the circuit variables, a control-oriented model in state space can be obtained. Define the state variable as $x=V_{c}$ (potential drop across $\left(C_{a}+C_{1}\right)$ ), input variable $u=V-V^{-}$and system output $y=H_{2}$, the system current responsible for gas production. A first order state space model is obtained where $\dot{x}$ is the differential equation for the circuit in Figure 3 and is given as:

$$
\begin{aligned}
\dot{x} & =-x \frac{1}{C_{1}+C_{a}} \frac{R_{a}+R_{c}+R_{d c}}{R_{d c}\left(R_{a}+R_{c}\right)}+\frac{u}{\left(C_{1}+C_{a}\right)\left(R_{a}+R_{c}\right)}, \\
y & =\frac{\alpha x}{R_{d c}} .
\end{aligned}
$$

\section{Model Verification and Linearization}

\subsection{Experimental Setup}

An experiment was set up to measure the flow rate of hydrogen gas at different input voltages. A one-membrane IPMC model constructed in lab was used for this work. The single cell model comprised of a Nafion 1110 (258 $\mu \mathrm{m}$ thick, DuPont) IPMC membrane with platinum (Pt) electrodes (anode and cathode), a water chamber for electrolysis and measuring tube to collect produced gases. As shown in Figure 5, the membrane was enveloped between two electrodes. The material used for terminal connection was a 
gold $(\mathrm{Au})$ foil. Assembly of the membrane and electrode was hold together by frame made from acrylic sheet. The whole assembly was enclosed in a water chamber and the electrode terminals were brought out using cables. Each electrode terminal was connected to the positive and negative terminal of a DC power supply to form anode and cathode respectively. Note that IPMC was a bidirectional component i.e. either side along its length can be used as anode or cathode. Experimental setup consisted of a electrolysis model and a programmable DC Voltage source. Observations were made on the same arrangement at temperatures between $20^{\circ} \mathrm{C}-62^{\circ} \mathrm{C}$. The $\mathrm{DC}$ source used in this experiment was a programmable bipolar power supply (Kepco BOP 20-10, 200W). Electrical measurements were recorded using dSpace 1104 controller board. The molecules of hydrogen gas accumulated on cathode and that of oxygen on anode were collected in measurement tubes by displacement of gas inside water.

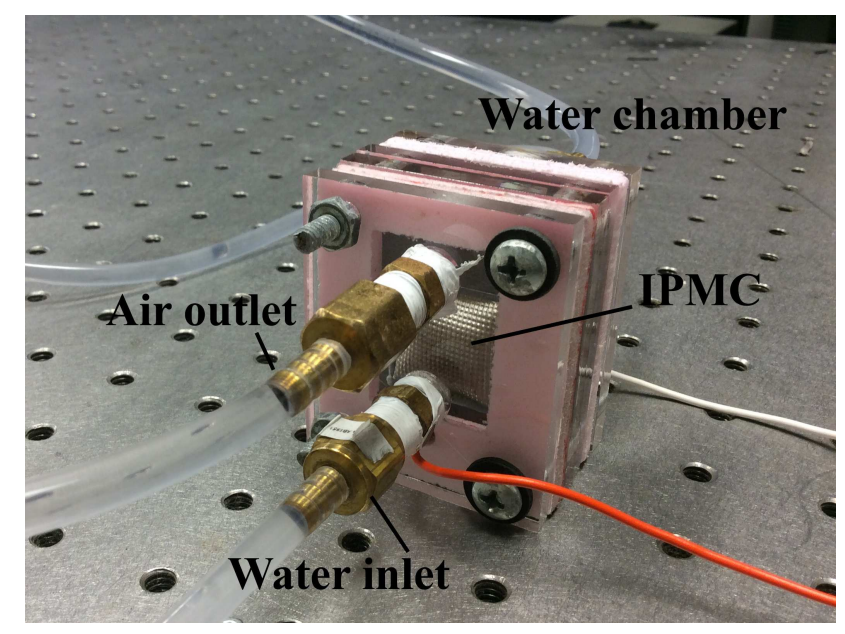

Figure 4: Test electrolyzer.

\subsection{Parameter identification}

The molar mass $(m)$, density $(\rho)$ of hydrogen and Faradays constant $(F)$ were constant values at a fixed absolute temperature. Since the model simulation was done at $300^{\circ} \mathrm{K}$, the electrical parameters were identified at a fixed absolute temperature and therefore did not require identification. Anion concentration $C^{-}$and dielectric constant $k_{e}$ were used based on the reported values in the literature [18].The remaining parameters were obtained by a 


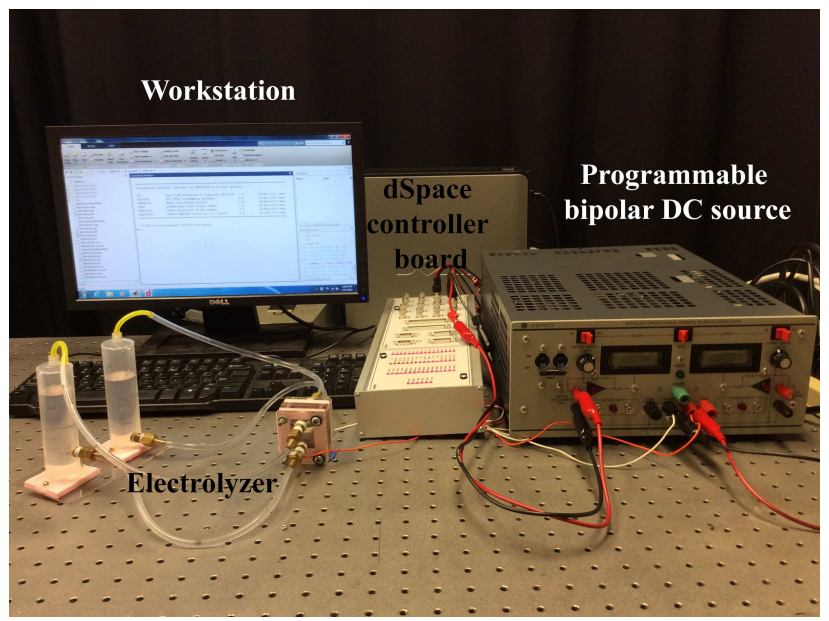

Figure 5: Experimental setup.

direct measurement, such as dimensions of IPMC membrane, circuit current and hydrogen gas generated. Under a series of voltage step inputs, the current responses of the membrane were measured. Measurement was taken for each step voltage input. In Figure 6, the initial value of source current $\left(I_{0}\right)$ was the current when capacitors had no charge on it, while $\left(I_{d c}\right)$ was the steady state value of circuit current, that was when capacitors were fully charged.

The electrode and ion diffusion resistance $\left(R_{a}+R_{c}\right)$ were obtained by calculating the slope of $I_{0}-V$ curve in Figure 6, because the initial current was the ratio of applied voltage to electrode and ion diffusion resistance.

$$
I_{0}=\frac{V}{R_{a}+R_{c}}
$$

The proportional gain $\alpha$ between the gas flow rate and source current was calculated based on the Equation (13). Given mass of hydrogen molecule $(m)$ as $0.00201588 \mathrm{~kg}$ per mole and density $(\rho)$ as $0.0899 \mathrm{~kg}$ per cubic meter at the temperature of $273^{\circ} \mathrm{K}, \alpha=7.5 \mathrm{~cm}^{3} / \mathrm{A}$. Based on Equation (29), the nonlinear DC conductance $G_{d c}$ was approximated by a third-order polynomial function whose coefficients can be identified by a nonlinear fitting process in Matlab. Figure 7 shows the results of nonlinear fitting process, which leads to the identified coefficients $m, n, o,, p$ shown in Table $1 . V^{-}$ was measured at the voltage when the DC current became none zero. 

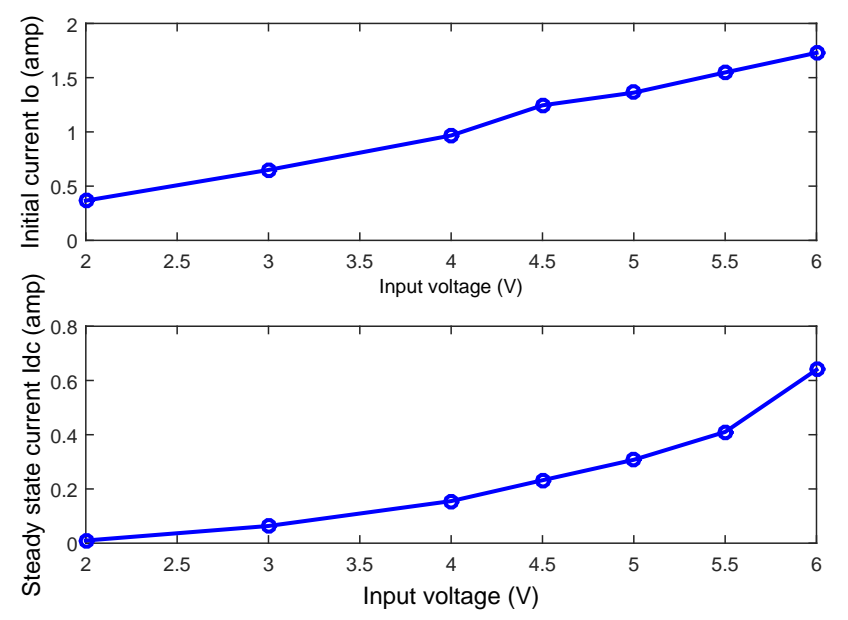

Figure 6: Initial current and steady state current versus voltage step.

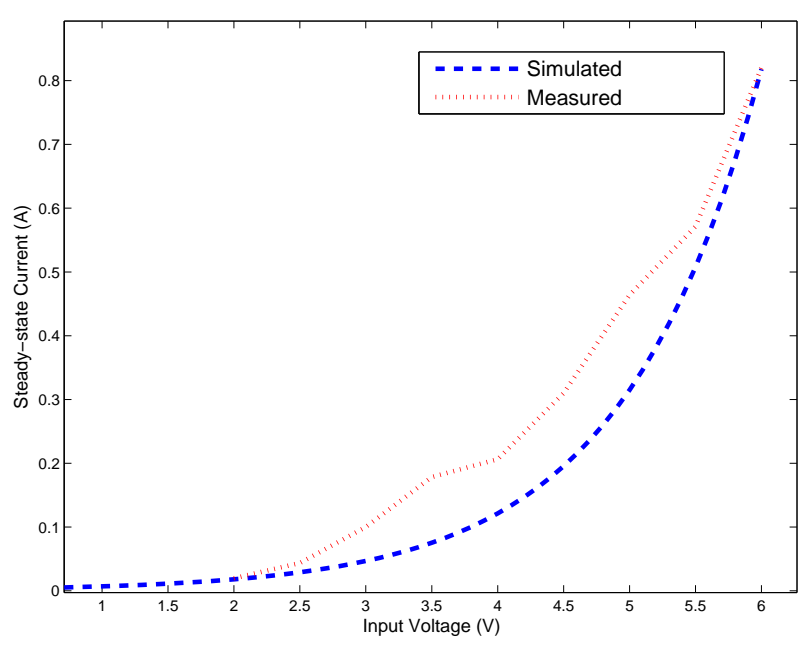

Figure 7: Nonlinear fitting of nonlinear conductance with a third-order polynomial function. 
Table 1: Parameter of IPMC membrane.

\begin{tabular}{|cc|cc|}
\hline$S$ & $6.25 \mathrm{~cm}^{2}$ & $R$ & $8.3143 \mathrm{~J} / \mathrm{mol}^{\circ} \mathrm{K}$ \\
\hline Thickness & $180 \mu \mathrm{m}$ & $T$ & $300{ }^{\circ} \mathrm{K}$ \\
\hline$C-$ & $1091 \mathrm{~mol} / \mathrm{m}^{3}$ & $k_{e}$ & $1.34 \times 10^{-6} \mathrm{~F} / \mathrm{m}$ \\
\hline$K$ & 7.5 & $c^{H^{+}}$ & $1 \times 10^{6} \mathrm{~mol} / \mathrm{m}^{3}$ \\
\hline$m$ & $-4.9195 \times 10^{3}$ & $n$ & $5.6049 \times 10^{3}$ \\
\hline$o$ & $-1.8613 \times 10^{3}$ & $p$ & $0.1860 \times 10^{3}$ \\
\hline$R_{a}+R_{c}$ & $5.44 \Omega$ & $V^{-}$ & $1.5 \mathrm{~V}$ \\
\hline
\end{tabular}

\subsection{Model validation}

The response of hydrogen gas flow rate under series of increasing voltage inputs was measured to realize the flow rate as observed in Equation (12). The corresponding input current to the system was measured as well. The test was done under room temperature $\left(20{ }^{\circ} \mathrm{C}\right)$ and standard atmospheric pressure $(1 \mathrm{~atm})$. Figure 8 shows graph of measured and calculated values of hydrogen gas flow rate for supply current $\left(I_{d c}\right)$. The theoretical curve of flow rate shows a slight deviation from the measured values. The possible reasons behind this could be measurement error, deviation in the electrical parameters or the theoretical curve represents steady state response, while the measured curve was due to dynamic conditions of the system.

The ohmic potential $\left(V_{\text {ohm }}\right)$ was obtained by current interrupt method explained in [22]. Under a steady-state condition, when the IPMC membrane capacitors were fully charged, the potential dropped across the circuit was due to $V_{\text {act }}$ and $V_{\text {ohm }}$. When the steady-state current to the system was interrupted, the potential drop across the electrode and ion diffusion resistance, that was the ohmic potential which reduced to zero. $V_{\mathrm{ohm}}=I\left(R_{a}+R_{c}\right)$, $I \rightarrow 0, V_{\mathrm{ohm}}=0$. Figure 9 shows an abrupt drop in the system voltage as system current went to zero. This difference in the voltage corresponded to the ohmic potential.

The obtained value of $\left(R_{a}+R_{c}\right)$, is verified using Equation (35). Figure 10 shows the comparison between the theoretical ohmic potential and the experimentally obtained values. It was observed from the figure that ohmic potential drop followed a linear relation with the steady-state current of the system. The actuation potential can be obtained using Equation (19), where $V_{\text {ohm }}$ was known from Equation (20) and $V^{-}$was considered constant at $300^{\circ} K$. The potential drop across capacitor branch was obtained from 


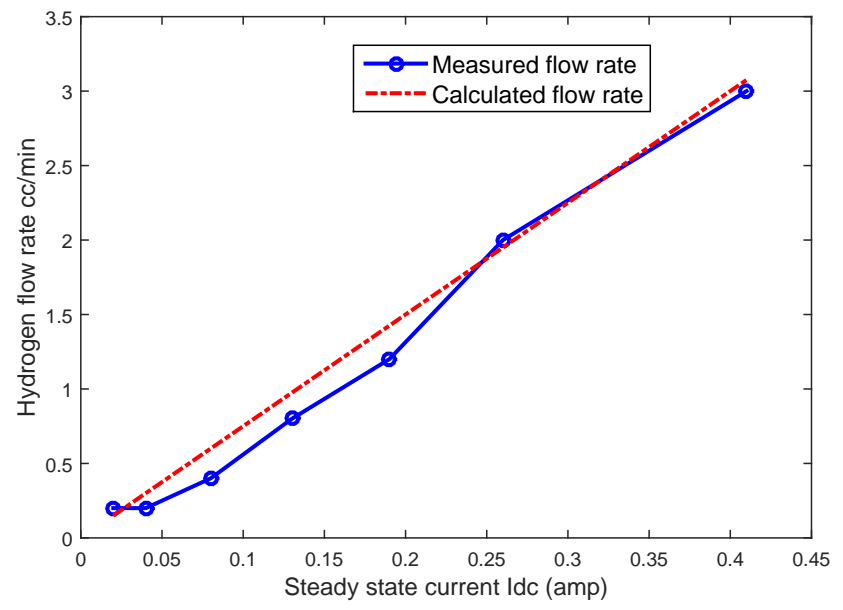

Figure 8: Measured and calculated flow rate of hydrogen gas for steady-state source current under room temperature $\left(20^{\circ} \mathrm{C}\right)$ and standard atmospheric pressure $(1 \mathrm{~atm})$.
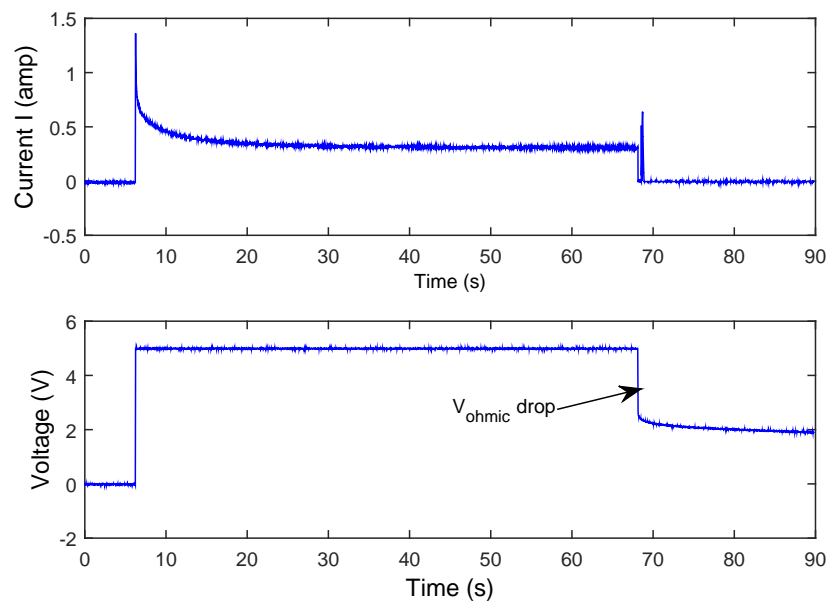

Figure 9: Ohmic potential drop. 
Equation (30). Then it was used to get a polynomial equation for nonlinear conductance $G_{d c}$.

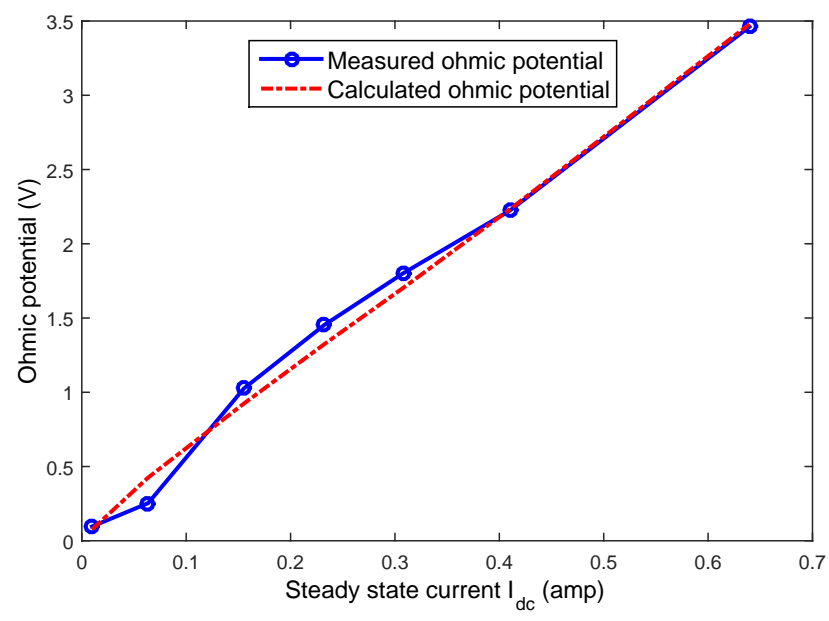

Figure 10: Steady-state current of IPMC electrolyzer versus ohmic potential drop.

\subsection{Model linearization}

The proposed physics-based model captured the non-linear characteristics of the system. The aim of this project was to design a linear quadratic regulator (LQR) controller to control the flow rate of hydrogen gas. The method started with defining state-space model and therefore it required linearization of the proposed non-linear model. To obtain linear approximation of Butler-Volmer equation (given in the Equation 29), the equation parameters were obtained using Tafel plot. The slope of Tafel plot defines the charge transfer coefficient and its $\mathrm{X}$ intercept, which gives the source current as

$$
I_{d c}=\frac{V_{\mathrm{act}}-V_{t h}}{R_{d c}^{\prime}},
$$

where $R_{d c}=1.584 \Omega$ is the linearized DC resistance and $V_{t h}=4.7 \mathrm{~V}$ is the threshold voltage. Figure 11 shows both the linearized DC resistance and nonlinear DC resistance. When the input voltage is larger than $5 \mathrm{~V}$, the model with linear DC resistance captures the experimental data well. The nonlinear capacitance $C_{1}(V)$ and $C_{2}(V)$ were calculated based on the 


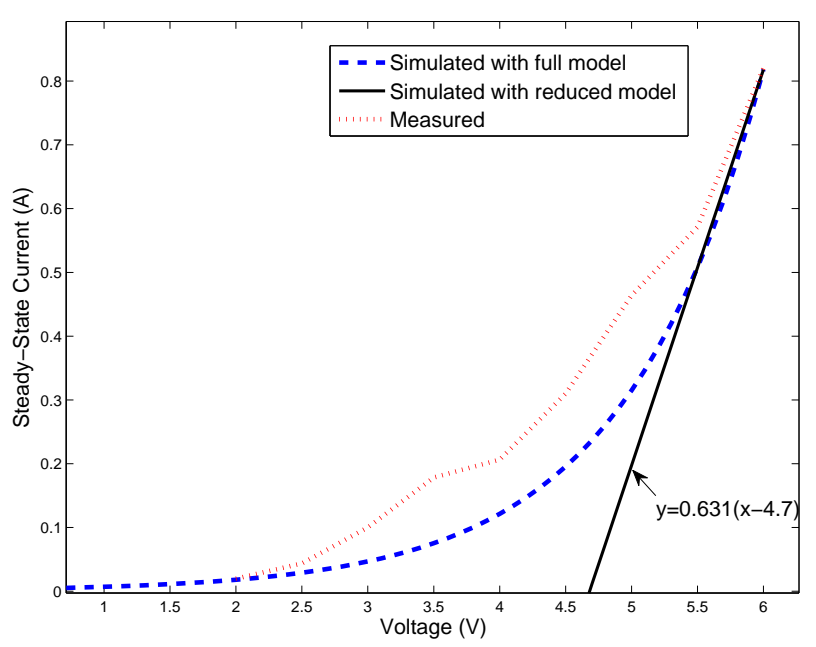

Figure 11: Linearized $R_{d c}$ resistance.

Equation (21) and (26) when $V=5$ volts which was the nominal value of operating voltage.

From Equation (12), it is evident that the output in terms of hydrogen gas flowrate was proportional to the source current. Therefore, the source current was the controlling factor for this system. A nonlinear first order state-space model was derived by linearizing the model (33) and (34) around the original point, which was

$$
\begin{aligned}
\dot{x} & =A x+B u \\
y & =C x+D u-2.967
\end{aligned}
$$

where

$$
A=-2.2009, B=3.76, C=0.631, D=0 .
$$

The proposed first order differential model can capture the transient and steady state response. Figure 12 shows the open-loop response of system current for a step input. Here the peak value of transient current and steady state current of the system were 1.362 and 0.3076 ampere respectively for a step input of 5 volts. 


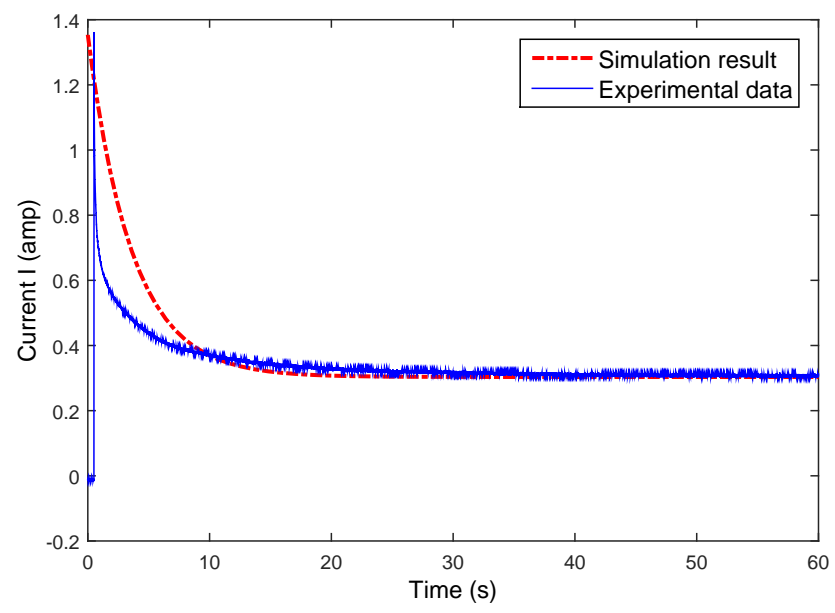

Figure 12: Open-loop step response.

\section{Investigation of Temperature dependent effect and energy con- version efficiency}

Experiments were carried out to understand the effect of temperature on IPMC electrolysis. With increase in the temperature of water, ion movement gained more energy. It resulted to decreasing in the resistance to the flow of ions into the polymer. Therefore, the current exchange density reduced and so did the Faradaic resistance. This resulted to increasing in the flow of current across membrane, as shown in Figure 13. With the increase in temperature, a smaller excitation leaded into a higher current. This was translated into a higher hydrogen production with less input power because, according to Equation (12), the flowrate of hydrogen gas was directly proportional to the source current.

The system efficiency was measured by observing the input energy and usable output energy from electrolysis. The volumetric output of hydrogen gas was measured and its stored energy was calculated by using the standard value of calorific content of hydrogen gas is $13000 \mathrm{~kJ} / \mathrm{m}^{3}$. The measured quantity is obtained in terms of kilojoules. The energy efficiency of the system is calculated by computing input energy to the system and the output energy from the measured calories. The energy-conversion efficiency can be calculated by

$$
E=\frac{V \rho \alpha / m}{U I_{d c} \Delta t}
$$




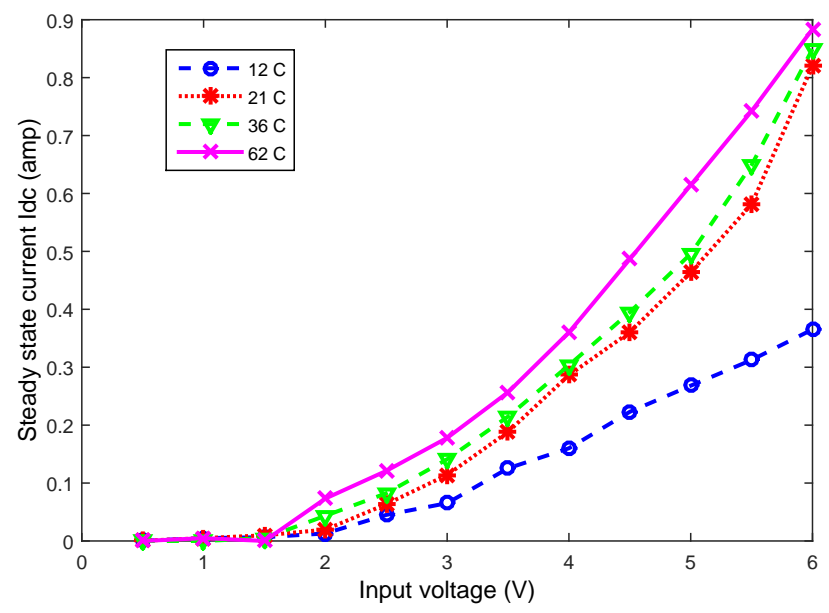

Figure 13: Current response of the system at different temperatures.

where $\Delta t$ is the duration time (second), $U$ is the applied DC voltage (volt), $I_{d c}$ is the source current (amp), $V$ is the measured volume of hydrogen gas (cc), $\rho$ is the density of hydrogen $(\mathrm{g} / \mathrm{cc}), m$ is the molar mass of hydrogen $(\mathrm{g} / \mathrm{mol}), \alpha_{\mathrm{HHV}}$ is the higher heat value (HHV) of hydrogen $(285.8 \mathrm{Joul} / \mathrm{mol})$. Figure 14 shows the system efficiency under a series of input voltages. It has been shown from the graph that the efficiency of the system decreased with the increase in the input power to the electrolyzer. Therefore it was necessary to control the applied voltage and the source current.

\section{Model-based Control Design and Implementation}

\subsection{LQR Control Design}

In order to achieve a better output performance, an optimal controller was designed. LQR is a method that minimizes the performance index of the system [23]. The performance index used in this project for LQR was:

$$
\begin{array}{r}
J(0)=\frac{1}{2} x(T)^{T} S(T) x(T)+ \\
\frac{1}{2} \int_{0}^{T} x(T)^{T} Q(T) x(T)+u(T)^{T} R(T) u(T) d t
\end{array}
$$

A standard LQR design approach was used to find optimal control input law $\left(u^{*}\right)$ such that under the constraints of $Q$ and $R$, which minimizes the 


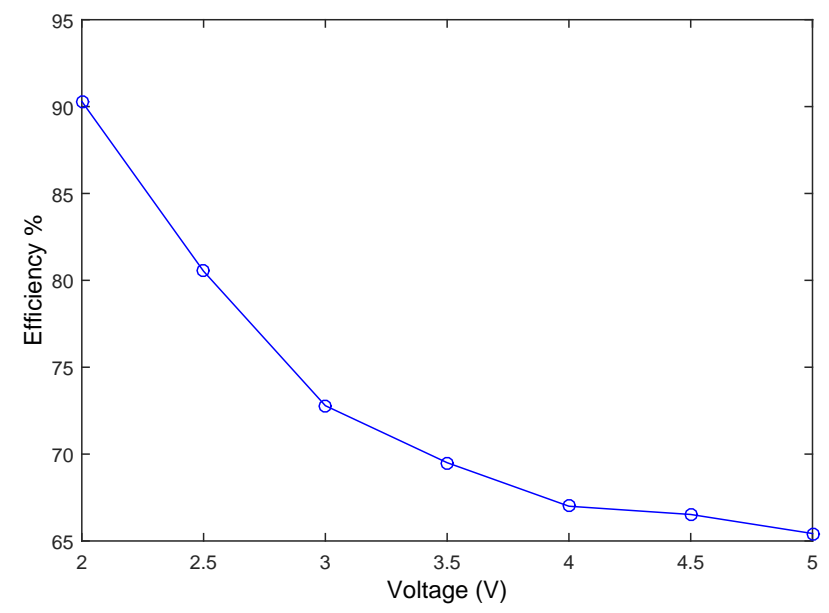

Figure 14: (Efficiency of the system versus voltage inputs.

performance index $(J(0))$. The optimal control law for close-loop was given as:

$$
u=-K x(t),
$$

where $K$ is the Kalman gain and determines the pole placement for minimum performance index for close-loop. It mainly depended on $A, B, Q$ and $R$ matrices. The equation to obtain $K$ was given as:

$$
\begin{gathered}
A^{T} S+S A-S B R^{-1} B^{T}=0 \\
K=R^{-1} B^{T} S
\end{gathered}
$$

For this controller the state $Q$ and control weighting $R$ matrix was obtained by trial and error. The simulation results were obtained for the proposed model between the performance of the controller and the reference model. The elements of state weighting matrix and the control weighting matrix were obtained to determine feedback gain $K$. The solution was for constant gain and optimum value of gain matrix $K$ obtained with given $Q=12$ and $R=0.6$ value is $K=5.276$. The system caught the reference at the final point in less than 25 seconds, which is shown in Figure 15(a). For a comparison purpose, a PID control was designed and implemented in real-time. The proportional, integral and derivative gain chosen for this model are $K_{p}=4.775, K_{i}=0.021$, and $K_{d}=0.001$. Figure $15(\mathrm{~b})$ shows a step response of the cell with PID controller. It can be observed that system 
settles accurately with the reference input. In real-time it is observed that the system entered into an oscillation between $-7 \mathrm{~V}$ and $+7 \mathrm{~V}$ with the derivative gain. Therefore no derivative gain was applied to the controller. It has been observed that the system took a longer time to settle with PID control. It was due to constraint on the maximum voltage of the system. The system is not allowed to exceed +7 volt.

\section{Conclusion and Future Work}

In this paper a nonlinear control-oriented model was proposed for IPMC as an electrolyzer. The model captures the electrochemical fundamentals of water electrolysis for hydrogen generation as well as ion transportation dynamics in IPMC. The electrochemical model shows that at constant temperature and pressure, the flow-rate of hydrogen is proportional to the steady state current drawn by the system. The internal parameters of IPMC electrolysis were realized and the system was represented in its electrical equivalent form. A state-space model was derived to verify the system response with the proposed model. Identification of the parameters was done through the literature survey and experiments. The system prediction error was less than $4.629 \%$. Experimental data have shown that the efficiency of the system decreased with increase in the input power and temperature variation affected the output performance. Therefore, a controller was designed to maintain the output flow-rate to maintain its optimum efficiency. Due to a slow settling response of the system with PID control, an LQR controller was designed based on optimal control theory. The controller was able to achieve a minimum settling time of 25 seconds, which was better than a model-free PID control.

IPMC exhibits electrical as well as mechanical hysteresis which can be considered as an uncertainty. The future work would be focused on hysteresis model of IPMC electrolysis to investigate the mechanical actuation effect on water electrolysis. The temperature-dependent effect on energy-conversion efficiency will also be investigated experimentally and theoretically.

\section{Acknowledgment}

This material is based upon work supported by the National Science Foundation under the Award No. EPS-0903806 and matching support from the State of Kansas through the Kansas Board of Regents. This work was 


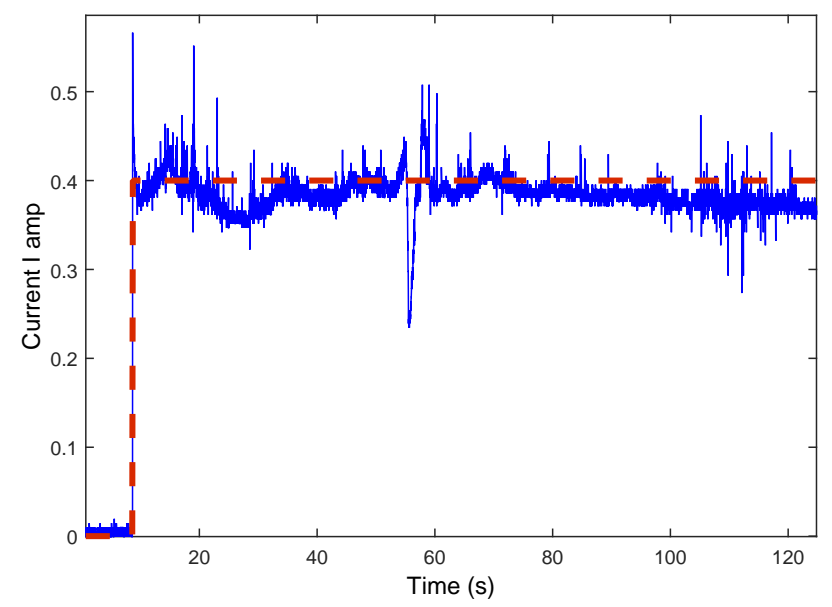

(a)

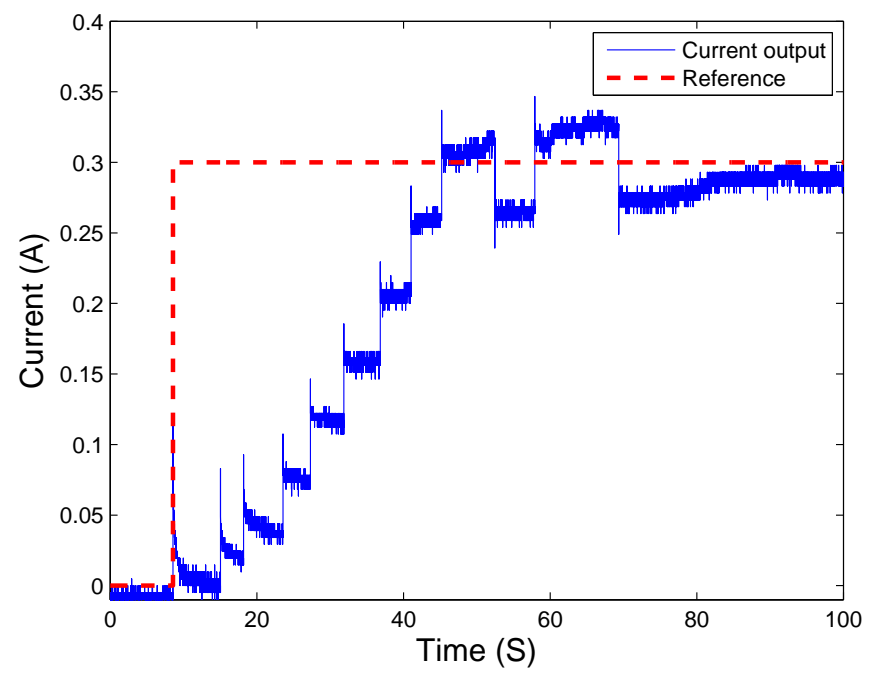

(b)

Figure 15: (a) A step response of the cell with LQR control; (b) a step response of the cell with PID control. 
also supported in part by the Award for Research and Creativity (ARC) Grant at Wichita State University and the Kansas Engineering Expansion Grant: House Substitute for Substitute Senate Bill No. 127, an act Enacting the University Engineering Initiative Act at Wichita State University.

[1] C.-J. Winter, Hydrogen energyabundant, efficient, clean: A debate over the energy-system-of-change, International Journal of Hydrogen Energy 34 (2009) S1S52.

[2] M. Ni, D. Y. Leung, M. K. Leung, A review on reforming bioethanol for hydrogen production, International Journal of Hydrogen Energy 32 (15) (2007) 3238e47.

[3] R. Chamousis, Overview of storage development doe hydrogen program, Sandia National Laboratories 9.

[4] Hydrogen production, http://energy.gov/eere/fuelcells/hydrogenproduction.

[5] M. Shahinpoor, K. Kim, Ionic polymer-metal composites: I. Fundamentals, Smart Materials and Structures 10 (2001) 819-833.

[6] M. Carmo, D. L. Fritz, J. Mergel, D. Stoltena, A comprehensive review on PEM water electrolysis, International Journal of Hydrogen Energy 38 (2013) 4901-4934.

[7] P. Millet, M. Pineri, R. Durand, New solid polymer electrolyte composites for water electrolysis, Journal of Applied Electrochemistry 19 (1989) $162-166$.

[8] K. J. Kim, M. Shahinpoor, A. Razani, Preparation of ipmes for use in fuel cells, electrolysis, and hydrogen sensors, in: Proc. of the SPIE Conference on Electroactive Polymer Actuators and Devices (EAPAD), 2000, pp. 311-320.

[9] J. Moore, C. Stanitski, P. Jurs, Principles of chemistry: the molecular science, Cengage Learning, 2009.

[10] J. Z. Zhang, J. Li, Y. Li, Y. Zhao, Hydrogen Generation, Storage and Utilization, John Wiley \& Sons,, 2014. 
[11] A. Shaaban, Pulsed DC and anode depolarization in water electrolysis for hydrogen generation, Tech. Rep. ESL-TR-92-55, AIR FORCE CIVIL ENGINEERING SUPPORT AGENCY TYNDALL AFB FL ENGINEERING AND SER VICES LAB (1994).

[12] N. Shimizu, S. Hotta, T. Sekiya, O. Oda, A novel method of hydrogen generation by water electrolysis using an ultra-short-pulse power supply, Journal of Applied Electrochemistry 36 (2006) 419-423.

[13] S. Nemat-Nasser, J. Li, Electromechanical response of ionic polymermetal composites, Journal of Applied Physics 87 (7) (2000) 3321-3331.

[14] K. M. Farinholt, Modeling and characterization of ionic polymer transducers for sensing and actuation, Ph.D. thesis, Virginia Polytechnic Institute and State University (2005).

[15] Z. Chen, X. Tan, A control-oriented, physics-based model for Ionic Polymer-Metal Composite actuators, in: Proceeding of IEEE 46th Control and Decision Conference, New Orland, 2007, pp. 590-595.

[16] B. E. Conway, Electrochemical Supercapacitors Scientific Fundamentals and Technological Applications, Springer, 1999.

[17] Z. Chen, D. R. Hedgepeth, X. Tan, A nonlinear, control-oriented model for ionic polymer-metal composite actuators, Smart Materials and Structures 18 (5) (2009) 055008.

[18] Z. Chen, D. Hedgepeth, X. Tan, A nonlinear control-oriented model for ionic polymer-metal composite actuators, in: Proceedings of the 47th IEEE Conference on Decision and Control, Cancun, Mexico, 2008, pp. $1851-1856$.

[19] B. J. Akle, Characterization and modeling of the ionomer-conductor interface in ionic polymer transducers, Ph.D. thesis, Virginia Polytechnic Institute and State University (2005).

[20] C. Lim, Reaction kinetics: Butler volmer equation, MIT Open Courseware.

[21] J. B. Jia, Y. T. Cham, The electrical dynamic response study of pemfc as a backup power supply, in: In Proc. of the IEEE International Conference on Control and Automation, 2007, pp. 1156-1161. 
[22] J. Larminie, Current interrupt techniques for circuit modelling, in: Electrochemical Measurement, IEE Colloquium on, 1994, pp. 12/1-12/6.

[23] F. L. Lewis, D. L. Vrabie, V. L. Syrmos, Optimal Control, John Wiley \& Sons, 2012. 